\title{
Unexplained dyspnea could be ascribable to postural changes
}

\author{
Eva Bernardi \\ Gaia Mandolesi \\ Luca Pomidori \\ Annalisa Cogo
}

Biomedical Sport Studies Center, University of Ferrara, Ferrara, Italy

\author{
Address for correspondence: \\ Eva Bernardi, MD \\ Biomedical Sport Studies Center \\ University of Ferrara \\ Via Gramicia 35 \\ 44100 Ferrara, Italy \\ Phone: +39 3470104401, +39 0532455829 \\ Fax: +39 0532705018 \\ E-mail: bernardi.eva@gmail.com
}

\section{Summary}

Here we present the cases of 3 females referred to our outpatient clinic complaining dyspnea during daily activity, in particular during walking. A careful history enabled us to determine that dyspnea was significantly reduced when they walked with upper-limb support.

Methods. Subjects underwent respiratory function tests (FEV1, FVC, DLCO), chest X-ray, echocardiogram with non-invasive PAPs measurement, blood sample testing and ventilatory evaluation by means of portable respiratory inductive plethysmography (LifeShirt System, LS). LS accurately estimates ventilation, ventilatory pattern and the synchrony of rib cage-abdominal motion (phase angle, $\mathrm{PhA}$ ) at rest and during exercise both in patients and in healthy subjects. Subjects performed six-minute walking tests (6MWT) with and without a rollator while equipped with LS.

Results. All exams were normal. LS evaluation during 6MWT showed an higher PhA during 6MWT without rollator: $26 \pm 11^{\circ}$ and $17 \pm 3^{\circ}$ without and with rollator respectively.

Conclusion. During 6MWT without upper-limb support an evident increase in PhA was observed. This is an indicator of rib cage-abdominal motion asynchrony, which in turn could induce dyspnea. We suggest that postural change during walking without upper-limb support may occur in other- wise elderly subjects and this could explain the dyspnea.

KEY WORDS: dyspnea, thoraco-abdominal kinematics, posture.

\section{Introduction}

Dyspnea is one of the symptoms that the pulmonary specialists most frequently encounter. Defined as "a subjective experience of breathing discomfort that consists of qualitatively distinct sensations that vary in intensity" (1), dyspnea is a distressing symptom that affects a large group of patients suffering from chronic diseases such as chronic obstructive pulmonary disease (COPD), asthma, heart failure, neuromuscular diseases, cancer, and is the main symptom of panic disorder (1). Breathlessness may also be associated with anxiety, fear and depression. This can contribute to the degree of disability associated with dyspnea, and the afflicted may avoid all activities likely to lead to such discomfort becoming steadily more sedentary and unfit. Hence, investigating the pathologic processes behind this symptom is essential as is finding effective forms of treatment.

\section{Clinical case}

Here we present the cases of three females (aged 7178) complaining of dyspnea during daily activity, in particular during walking, referred to our outpatient clinic. Constructing a careful history enabled us to determine that dyspnea was significantly reduced when they walked with the aid of upper-limb support.

Medical history and physical evaluation were normal, although two of the subjects were overweight (BMI 24, $26,30 \mathrm{~kg} / \mathrm{m}^{2}$ ). None of the subjects were taking any medications that could alter dyspnea perception (e.g. opioids).

Subjects performed a complete respiratory function test (PFT) including lung carbon monoxide diffusing capacity (DLCO), which showed no abnormalities. The chest X-ray was normal. In line with American Thoracic Society (ATS) statement (1), EKG and echocardiogram with non-invasive measurement of pulmonary arterial pressure were performed, and no anomalies were detected. Cardiological examination showed no cardiovascular abnormalities. A blood sample was taken and analysed for haematocrit, haemoglobin, iron profile and B-type natriuretic peptide (BNP), which were all within the normal range. 


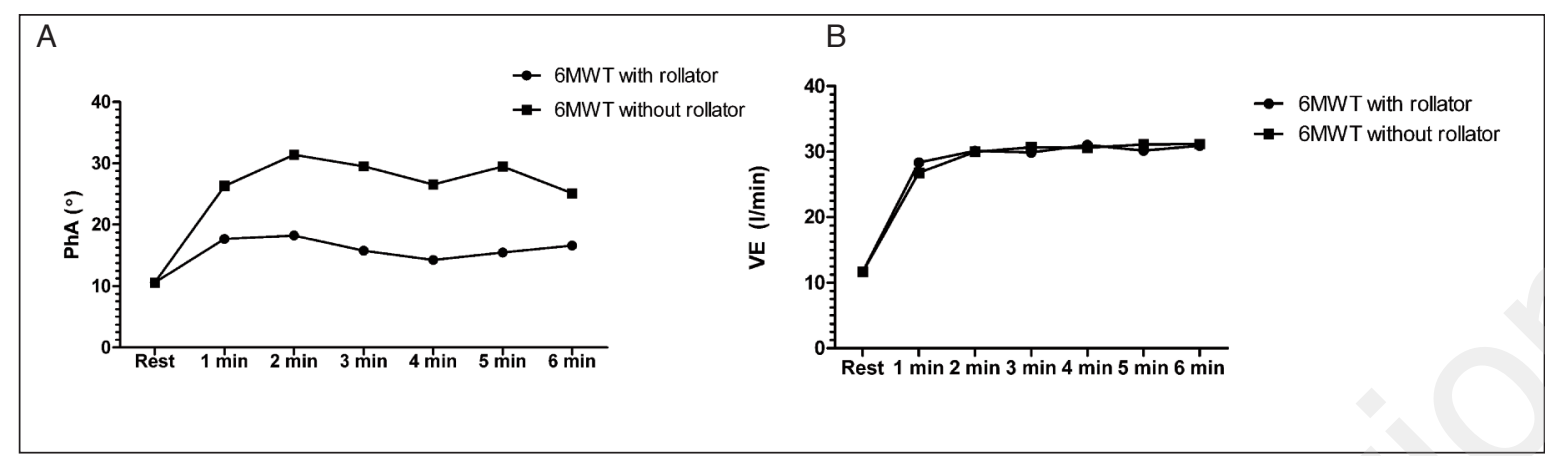

Figure 1 - The trend in phase angle (PhA, Panel A) and ventilation (VE, Panel B) during the 6 minute walking tests.

We then set out to identify other non-cardiorespiratory causes of exercise limitation (e.g. leg discomfort, fatigue, weakness), often associated with breathing discomfort by evaluating the patients' exercise capacity. Moreover, also neuromuscular disorders were excluded. Ventilation, ventilatory pattern, and the synchrony of rib cage-abdominal motion were assessed by a noninvasive portable inductive plethysmography (LifeShirt Systems, Vivometrics, California) during a six-minute walking test (6MWT). This device has been shown to indirectly but accurately estimate these parameters at rest and during exercise both in patients and in healthy subjects (2).

Subjects were familiarized with the test and then performed a 6MWT without a upper-limb support of (T1) in a $23 \mathrm{~m}$ long corridor. After 30 minutes of rest they performed another 6MWT, this time with the aid of a rollator (T2). The same standard phrases were used to encourage the patients in both tests. Dyspnea was assessed at rest and during both exercise tests using the Borg Scale, a sensory-perceptual measure of what breathing "feels like" to the patients who scored the intensity of their discomfort. The patients' perceived exertion was scored every minute.

The distance covered in each tests was identical. Likewise, analysis of ventilation and breathing pattern revealed no differences between aided and unaided walking: ventilation (T1: $29.4 \pm 2.9 \mathrm{l} / \mathrm{min}, \mathrm{T} 2: 29.4 \pm$ $6.3 \mathrm{l} / \mathrm{min})$, respiratory rate $(\mathrm{T} 1: 28 \pm 4 \mathrm{~b} / \mathrm{min}, \mathrm{T} 2: 29 \pm$ $4 \mathrm{~b} / \mathrm{min})$ and tidal volume (T1: $1.09 \pm 0.18 \mathrm{I}, \mathrm{T} 2: 1.04$ \pm 0.23 I). No oxygen desaturation was recorded. The only data that significantly changed between aided and unaided was the phase angle (PhA). PhA is an indicator of coordination between the rib cage and abdominal movements and an increase in this parameter is a signal of deteriorating coordination. At rest, in standing position, the average $\mathrm{PhA}$ was $10.8^{\circ} \pm 2.1$. Analysis of PhA trend during 6MWT showed a higher value during walking without the rollator (2-way ANOVA, $P=0.003)$ : in fact the average PhA in $\mathrm{T} 1$ was 26.1 $\pm 11.3^{\circ}$ and in $\mathrm{T} 2$ it was $16.9 \pm 3.3^{\circ}$.

The Borg score of dyspnea at the end of 6MWT was $8 / 10$ in $\mathrm{T} 1$ and $5 / 10$ in $\mathrm{T} 2$.

The trend in ventilation and PhA during 6MWTs is reported in Figure 1.

\section{Discussion}

In these three subjects with unexplained dyspnea we found a deterioration in thoraco-abdominal coordination during walking without upper-limb support. During quiet breathing, in the standing posture, the movements of the abdomen and the rib

The phase angle $(\mathrm{PhA})$ is an indicator of coordination between the rib cage and abdominal movements. cage are simultaneously coordinated and the observed increase in PhA during exercise is an indicator of asynchrony between the rib cage and abdominal compartments. This finding is associated with a higher Borg dyspnea score. In fact a major distortion of the basal thoraco-abdominal configuration is correlated with a greater elastic work (3), which in turn could induce dyspnea. Up to now there has been a paucity of data regarding the relationship between change in posture and dyspnea in the literature, although studies into this matter are becoming more common. Recently it has been shown that posture can influence thoraco-abdominal kinematics; in fact Romei et al. (4) showed that progressive inclination of the trunk induces a progressive reduction of rib cage displacement in healthy subjects. Furthermore, Chien JY et al. (5) have demonstrated that tho-

Postural changes in otherwise healthy elderly subjects can induce rib-cage-abdominal asynchrony during walking without upper-limb support resulting a greater elastic work, which can induce dyspnea. raco-abdominal asynchrony could contribute to exercise limitation in COPD patients predicting poor walking capacity, although the underlying mechanism is still unclear.

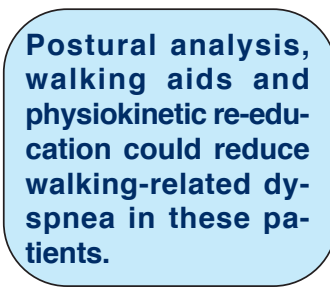

Although we did not analyse posture in our subjects, we were able to observe their chest position during the 6MWTs, and a forward inclination of the trunk was evident during unaided walking. In future our hypothesis 
should be confirmed by a postural analysis during walking.

In conclusion, we suggest that postural change during walking without upper-limb support can occur in the elderly and this could explain dyspnea in otherwise healthy subjects. More studies are needed to further investigate the link between rib cage-abdominal asynchrony and postural change and its impact on exercise dyspnea. We emphasised that all test results are in the normal range in only few of the subjects referred to outpatient clinics for dyspnea. These cases highlight the importance of thinking beyond the usual tests when evaluating subjects with dyspnea and indicate the importance of posture examination in the elderly. If our hypothesis that postural change can induce rib cage-abdominal asynchrony is confirmed, postural analysis, walking aids and re-education could help reduce walking-related dyspnea in otherwise healthy elderly patients.

\section{Disclosure}

This study had not financial support. The Authors declare no conflicts of interest.

\section{References}

1. Parshall MB, Schwartzstein RM, Adams L, Banzett RB, Manning HL, Bourbeau J, et al. American Thoracic Society Committee on Dyspnea. An official American Thoracic Society statement: update on the mechanisms, assessment, and management of dyspnea. Am J Respir Crit Care Med. 2012;185 (4):435-52.

2. Clarenbach CF, Senn O, Brack T, Senn O, Noll G, Russi EW, et al. Monitoring of ventilation during exercise by a portable respiratory inductive plethysmograph. Chest. 2005;128:1282-1290.

3. Goldman MD, Grimby G, Mead J. Mechanical work of breathing derived from rib cage and abdominal VP partitioning. JAP. 1976;41(5):75263.

4. Romei M, Mauro AL, D'Angelo MG, Turconi AC, Bresolin N, Pedotti A, et al. Effects of gender and posture on thoraco-abdominal kinematics during quiet breathing in healthy adults. Respir Physiol Neurobiol. 2010;172(3):184-91.

5. Chien JY, Ruan SY, Huang YCT, Yu CJ, Yang PC. Asyncronous thoraco-abdominal motion contributes to decreased 6-minute walk test in patients with COPD. Respir Care. 2013;58(2):320-326. 